\title{
9.4-14.1 T small-animal PET-MR imaging: Feasibility analysis of LYSO APD readout via long signal lines
}

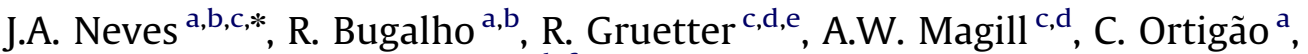 \\ J.C. Silva ${ }^{a}$, R. Silva ${ }^{\mathrm{a}}$, J. Varela ${ }^{\mathrm{a}, \mathrm{b}, \mathrm{f}}$ \\ ${ }^{a}$ LIP - Laboratory of Instrumentation and Experimental Particle Physics, Lisbon, Portugal \\ ${ }^{\mathrm{b}}$ IST-UTL - Instituto Superior Técnico, Technical University of Lisbon, Lisbon, Portugal \\ ${ }^{\mathrm{c}}$ Laboratory of Functional and Metabolic Imaging, École Polytechnique Fédérale de Lausanne, Lausanne, Switzerland \\ ${ }^{\mathrm{d}}$ Department of Radiology, University of Lausanne, Lausanne, Switzerland \\ e Department of Radiology, University of Geneva, Geneva, Switzerland \\ ${ }^{\mathrm{f}}$ CERN - European Organization for Nuclear Research, Geneva, Switzerland
}

\section{A R T I C L E I N F O}

Available online 23 August 2012

Keywords:

Small-animal PET

ClearPEM

UHF-MRI

Simultaneous PET-MRI

\begin{abstract}
A B S T R A C T
In the present work we intend to assess the readout feasibility of Avalanche Photodiode (APD) detectors via long signal lines for the development of a combined small-animal PET-MR prototype based on the ClearPEM technology. The detection performance of a LYSO-APD module was evaluated reading out the APD charge signals to the front-end ASIC via an $80 \mathrm{~mm}$ length flexible flat-cable (FFC). Experimental results showed a time resolution of $5.06 \mathrm{~ns}$ for the detector module in double-readout mode, with a nonsignificant degradation of $8.4 \%$ with the introduction of the FFC. The energy resolution of the system was not degradated by the FFC.
\end{abstract}

(c) 2012 Elsevier B.V. All rights reserved.

\section{Introduction}

The development and integration of new MR-compatible PET detectors within ultra-high field (UHF) magnets have been subject of increased interest in the scientific community. In this context, a new approach of the ClearPEM detectors and front-end electronics have been addressed for simultaneous small-animal PET-MR imaging in the 9.4 T and 14.1 T Varian magnets available at EPFL, Lausanne, Switzerland.

The ClearPEM is a dedicated APD-based PET detector for highresolution breast cancer imaging [1]. The basic detector supermodule is composed of 12 modules of $4 \times 8$ LYSO:Ce crystal matrices optically coupled to Hamamatsu S8550 APD arrays on both ends, and assembled between two front-end electronic boards (FEB). Each FEB integrates two low-noise ASICs for the readout of 192 channels, pulse amplification and shaping, performing also sequentially analog-to-digital conversion and data serialization and transmission to the off-detector data acquisition system [2]. Previous studies in the $7 \mathrm{~T}$ magnet facility at EPFL have demonstrated the mutual electromagnetic interference mechanisms and the integration feasibility of the ClearPEM front-end electronics and the MR system components [3].

\footnotetext{
* Corresponding author at: LIP - Laboratory of Instrumentation and Experimental Particle Physics, Lisbon, Portugal. Tel.: +351960 039281.

E-mail address: janeves@lip.pt (J.A. Neves).
}

The development of a small-animal PET insert based on ClearPEM technology implies a new design of the detector head to fit into the tightly constrained bore of UHF magnets. The system design proposal consists of 24 detector modules arranged in a ring geometry $(22.4 \mathrm{~mm}$ axial FoV $) .{ }^{1}$ Each module is composed of a matrix of $4 \times 8$ LYSO crystals $\left(2 \times 2 \times L \mathrm{~mm}^{3}\right.$ individual dimensions, with $L \in[10-15] \mathrm{mm}$ ) optically coupled to an $\mathrm{S} 8550$ APD array on the external readout side. The APD charge signals from each detector module are transmitted to the front-end ASICs via a flexible flat-cable (FFC) made of 32 APD signaling lines and 2 HV bias routed in a stripline configuration between two copper planes for EMI minimization, see Fig. 1.

In the proposed system design two front-end electronic boards are placed back-to-black longitudinally after the detector ring, receiving the $24 \mathrm{FFC}$ (with a maximum length up to $180 \mathrm{~mm}$ and bending up to $90^{\circ}$ ) from the detector modules. With this configuration, the need for APD cooling is avoided and the front-end boards are kept away from the magnet isocenter reducing the magnetic susceptibility interference artifacts caused by PCB materials in the spherical volume of the scanner. Both electronic boards will be sharing the same water-cooling plate, and together with the detector modules and the FFCs will be enclosed into a

\footnotetext{
${ }^{1}$ With this configuration the internal diameter of the ring is $85 \mathrm{~mm}$, leaving enough space for the RF coil and a small-animal (rodents) but restricting the space for PET components (the external diameter should not exceed $120 \mathrm{~mm}$ or $140 \mathrm{~mm}$, respectively for the $14.1 \mathrm{~T}$ and $9.4 \mathrm{~T}$ magnets).
} 


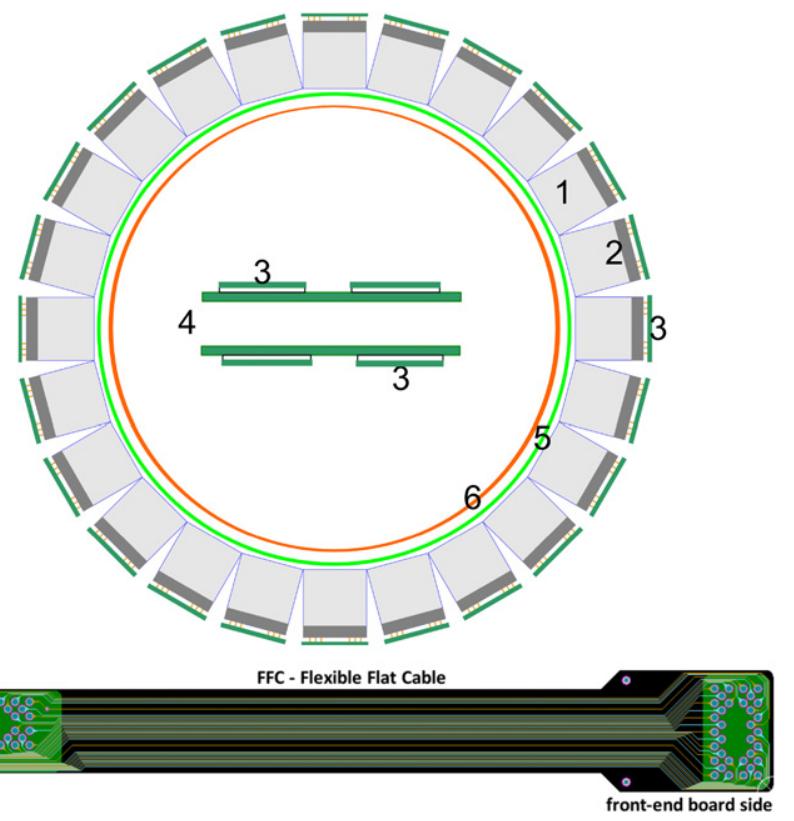

Fig. 1. Schematic drawing representing the system design proposal for the smallanimal PET detector ring: 1. the LYSO:Ce crystal matrices; 2. Hamamatsu S8550 $4 \times 8$ pixels ADP arrays; 3 . flexible flat-cables (APD connection and front-end side); 4. front-end readout boards; 5 . internal RF shielding cage (copper); 6. RF coil. The layout of the proposed FFC is shown in the bottom figure.

cylindrical RF shielding cage. Proper shielded cables will be used for Power (LV, HV) and Signals (Clock, Control, Data) to the PSUs and DAQ system placed externally to the magnet room.

In the present work we intend to assess the feasibility of APD readout via long signaling lines for the development of a combined small-animal PET-MR prototype.

\section{Materials and methods}

In order to evaluate the feasibility of APD readout via long signaling lines, a Lab-made $80 \mathrm{~mm}$ length FFC test version was produced from a flat ribbon cable $\left(0.5 \mathrm{~mm}\right.$ pitch, $0.081 \mathrm{~mm}^{2}$ cross-section) for a total of 32 APD signaling lines and $2 \mathrm{HV}$ bias. The FFC was shielded to minimize external electromagnetic noise pickup between the detector module and the front-end ASIC.

The effects of the FFC on detector timing performance were evaluated by the analysis of acquired data from a ClearPEM detector module ( $4 \times 8$ LYSO crystal matrix) assembled between two APD arrays in the conventional double-readout mode, one of the LYSO:APD readout sides being connected to the front-end ASIC through the FFC. To assess the FFC effects on the energy discrimination performance, two detector modules were assembled in single-side readout mode and positioned front to front at a distance of $73 \mathrm{~mm}$ for coincidence measurements with a ${ }^{22} \mathrm{Na}$ point source located at the center of the system (an energy calibration was performed acquiring data with different energy radioactive sources). The readout performance was compared by acquiring data with and without the FFC between the detector modules and the front-end ASICs.

\section{Experimental results and discussion}

\subsection{Timing performance}

Fig. 2 shows a typical profile histogram from the pulse samples of one LYSO:APD channel corresponding to the reconstructed

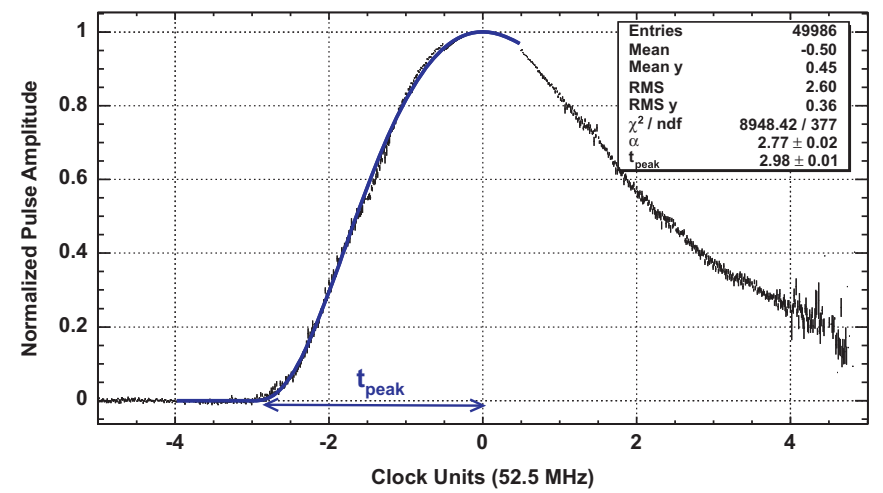

Fig. 2. Profile histogram of pulse samples from one LYSO:APD channel after the timing calibration process. Pulse rise time (10-90\% peaking time $\left|t_{\text {peak }}\right|$ ) is extracted from a parameterized function fitted to the data (blue solid line). (For interpretation of the references to color in this figure caption, the reader is referred to the web version of this article.)

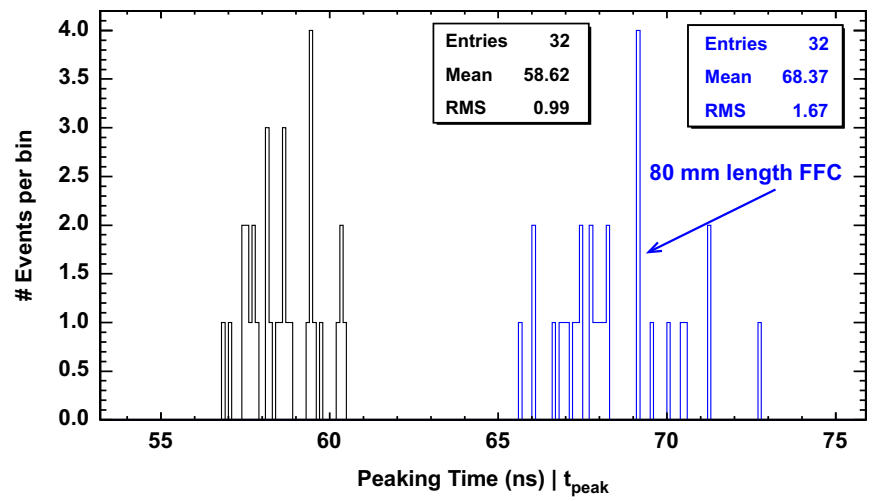

Fig. 3. Peaking time distribution for each block of 32 LYSO:APD channels (blue ones corresponding to those channels with the FFC). (For interpretation of the references to color in this figure caption, the reader is referred to the web version of this article.)

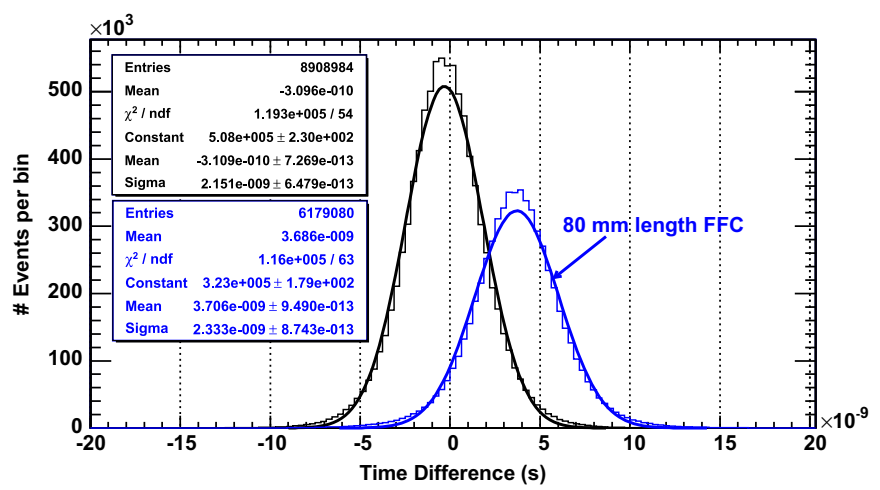

Fig. 4. Non-normalized time difference spectra showing the timing performance degradation with the FFC introduction in one LYSO:APD readout side.

shape of the signals from that channel. Data acquisitions in double-readout mode using a FFC in one of the sides have shown an average increase of the signal rise time of $7.8 \mathrm{~ns}$. The noncontrolled capacitance/inductance of the FFC propagation line is responsible for the observed spreading and shift of the peaking time histograms corresponding to the 32 channels $(4 \times 8$ crystal matrix) where the FFC was introduced, see Fig. 3.

The timing resolution of the system was measured as the FWHM of the time difference spectrum from events in the same crystal acquired within a 20 ns time window on the top and 


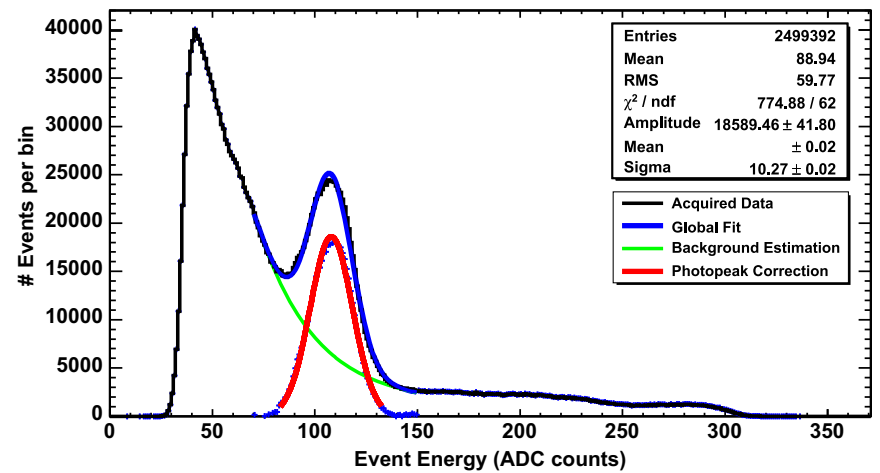

Fig. 5. Energy spectrum in single-side readout mode for a ${ }^{22} \mathrm{Na}$ point source (511 keV photopeak).

bottom APD readout channels. Fig. 4 presents a timing resolution of $5.06 \mathrm{~ns}$ FWHM $(2.35 \sigma)$ for the detector module in doublereadout mode (black line, compatible with ClearPEM results [1]), and a timing resolution degradation of $8.4 \%$ (5.49 ns FWHM) with the introduction of the FFC which is also responsible for a mean signal propagation delay of $3.7 \mathrm{~ns}$.

\subsection{Energy detection performance}

Acquired data from two detector modules in a coincidence detection scheme (LYSO:APD single-side readout mode) with a ${ }^{22} \mathrm{Na}$ point source have shown an energy resolution of $22.4 \%$ FWHM for $511 \mathrm{keV}$ photons (Fig. 5) and a good agreement with the expected linear energy calibration (Fig. 6). No energy performance degradation was observed in the measurements with the FFC introduction. The lower observed energy resolution compared with the ClearPEM system is due to the fact that the LYSO crystals are not optimized for being read out in single-ended mode (having one lateral face unpolished to spread scintillation light to both ends). In single-side readout mode this contributes to light losses and energy resolution degradation.

In conclusion, preliminary measurements with a Lab-made FFC of $80 \mathrm{~mm}$ length have shown nonsignificant degradation on

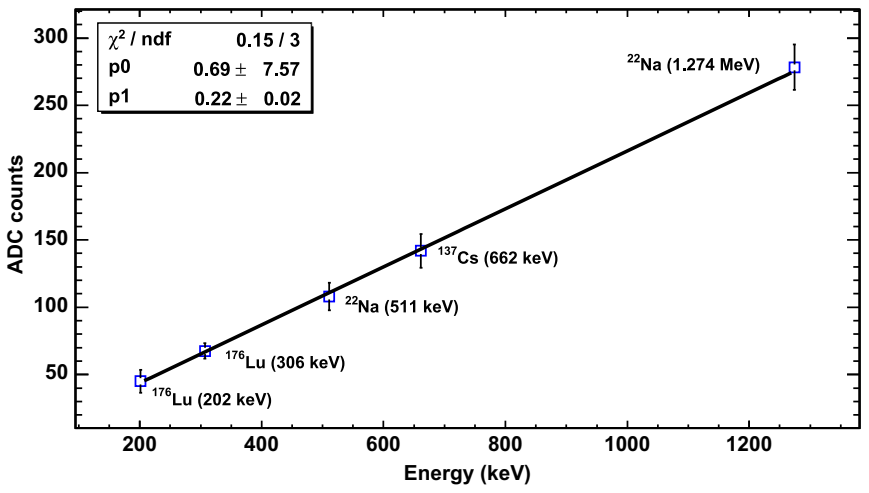

Fig. 6. Energy calibration in single-side readout mode: linear relation between the photopeak position (in ADC counts) and the equivalent energy (in keV) measured from different radiation sources, ${ }^{176} \mathrm{Lu}$ (LYSO:Ce), ${ }^{22} \mathrm{Na}$ and ${ }^{137} \mathrm{Cs}$.

the time and energy resolution demonstrating the feasibility of this new approach of the ClearPEM technology for the development of a small-animal PET insert for UHF magnets.

\section{Acknowledgments}

The author's work is supported by FCT, Portuguese Foundation for Science and Technology, under the Grant SFRH/BD/33667/ 2009. The author would also like to thank colleagues from LIP and CIBM-EPFL for their contribution.

\section{References}

[1] J.A. Neves, Nuclear Instruments and Methods in Physics Research Section A 628 (2011) 444.

[2] E. Albuquerque, et al., Nuclear Instruments and Methods in Physics Research Section A 598 (2009) 802.

[3] J.A. Neves, Feasibility and electromagnetic compatibility study of the ClearPEM frontend electronics for simultaneous PET-MR imaging, Presented at the PSMR2012 Conference, Isola d'Elba, Italy, 26-30 May 2012. 